\title{
Optimization of the layout of neutral sections based on a differential evolution algorithm
}

\author{
S. B. Song, P. F. Sun \& Q. Y. Wang \\ Southwest Jiaotong University, China
}

\begin{abstract}
This paper researches optimizing schemes of the setting of neutral sections in order to minimize the delayed time that they cause. Local optimization rules are first put forward through the model of a train passing over a neutral section. Quantitative analyses indicate that the neutral section should be set in a section where the train runs at a constant speed of $150-300 \mathrm{~km} / \mathrm{h}$ when passing over a neutral section. A differential evolution algorithm (DE) is then introduced to realize global optimization for the layouts of neutral sections along a whole railway line. What's more, a simulation platform is developed, which can simulate the train's operation off-line and obtain the optimal layout scheme for neutral sections on a given railway line. Finally, a case study of the BeijingShanghai high-speed railway line is conducted on the basis of a simulation platform. Simulation results show that, with the optimal neutral section setting scheme, a train's running time is reduced by 372 seconds in comparison with the original neutral section setting scheme.

Keywords: neutral section, layout optimization, differential evolution algorithm, simulation platform.
\end{abstract}

\section{Introduction}

With the rapid development of high-speed railways (HSR) in China, scientific planning has been taken into consideration in the HSR design. In the early stages of HSR design, operation safety has always been considered as fundamental, and the improvement of operation capacity as well as the decreasing of transportation cost as optimization objects. Under this circumstance, the layout of neutral sections begins to draw more and more attention on how to influence a train's speed and its running time. Improper neutral section settings could reduce the 
average speed of a train or even greatly restrict the train to reach the railway maximum allowable speed. Meanwhile, improper neutral section settings could also raise the construction and maintenance cost of railway lines and challenge the security of train operation. As a result, it is of great importance for electrified railways to select reasonable neutral sections' layout schemes.

For a long time, a train's running speed was generally low in China. The delayed time caused by neutral sections was not the main reason to restrain a train's running speed. Therefore, only a few studies has been done on train operation when passing through a neutral section considering train control, line conditions and global optimization of neutral sections' layout. In the literature [1], factors affecting velocity and time loss of the electric locomotive are discussed when passing over a neutral section. In addition, research is conducted on how the characteristics of an electric locomotive, the route conditions and length of a neutral section influence a train's running state. In the literature [2], the process of a train passing over a neutral section is analyzed, the mathematical model of the process is then established and rules of a single neutral section's layout for HSR are finally put forward. The relationship is firstly analyzed between train speed and its running time when passing over a neutral section and a genetic algorithm is finally applied to realize global optimization for neutral sections' layout scheme [3]. In literature [1, 2] and [4-6], research is aimed at a single neutral section, which deeply analyzes a train's operation running over a neutral section. In addition, recommendations are given on a single neutral section's layout. But these studies don't realize global optimization of the layout of neutral sections on a whole railway line or give constructive guidance for the existing HSR's reconstruction. Therefore, it is essential to research the motion mechanism and electrical process of a train when passing over a neutral section, mainly focusing on how a neutral section and the train's entering speed influence the train's operation.

Based on theoretical study of a neutral section's layout [3] and existing train traction calculation software [7], studies are done in this paper on optimizing schemes of neutral section settings. Local optimization rules are first put forward through the model of a train passing over a neutral section. A differential evolution algorithm is then introduced to realize global optimization for the layout of neutral sections on a whole line. Finally, a case study of BeijingShanghai high-speed line is conducted. Simulation results show that, with the optimal neutral section setting scheme, a train's running time is reduced compared with the original neutral section setting scheme.

\section{Automatic neutral section passing with the power off}

\subsection{Process description of passing over a neutral section}

The neutral section is a very important element on the railway to ensure mechanical connection and electric isolation among different electric phases. Nowadays, it's the articulated neutral section that is commonly adopted on the HSR in China. There are two modes of passing over a neutral section for electric 
multiple units (EMU), which are with the power on and off [2]. The mode of passing over a neutral section with the power on mainly depends on the ground switches of the neutral section, usually with the power off being less than $0.5 \mathrm{~s}$. Due to the high price of this type of ground equipment and its complex maintenance, this mode of passing a neutral section is rarely adopted domestically. In China, the EMUs pass through neutral sections with the electricity cut according to instructions pre-given by the signal system. Figure 1 shows the process of auto-passing a neutral section of an EMU.

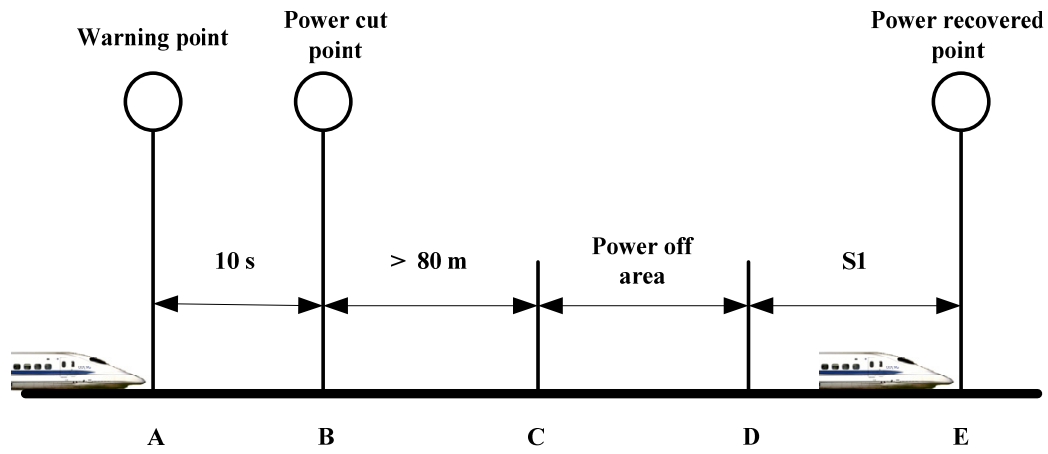

Figure 1: The process of auto-passing a neutral section of an EMU.

The process of passing through a neutral section of an EMU can be described as follows: the EMU receives warning information about the neutral section ahead; twice from two sets of balises respectively; A-B, after receiving warning information for the third time, the EMU continues running for about 10 seconds; $\mathrm{B}-\mathrm{C}$, on-board equipment of the EMU will be degraded, and the main circuit breaker is turned off; $\mathrm{C}-\mathrm{E}$, the EMU coasts until reaching the power recovered point; After passing over point $\mathrm{E}$, the main circuit breaker of the EMU will be turned on and the EMU may run with its maximum traction ability. Section D-E (S1) is the running distance during the reaction time of the on-board equipment after receiving the instruction to turn on the main circuit breaker, which differs according to different types of EMU. Section B-E is a whole process of a train passing through a neutral section. The EMU loses traction force after entering the neutral section with the power off. When the EMU remains in the neutral section, only resistance force is applied to the EMU, possibly as well as air brake force. Therefore, the train's speed may decrease with its running time increased. Since the speed of a train decreases after passing over the neutral section, there will definitely be an influenced section after the neutral section, which is also researched and defined as the chasing section in this paper.

\subsection{Mathematical modelling of passing over a neutral section}

The train running process depends on adhesion traction on the railway line, which leads to the train's acceleration. Considering each carriage of the train as a 
particle, a multi-particle model is then established. When passing through a neutral section, the train loses traction force. Therefore, assuming that the length of the ramp is far greater than the length of the train, according to Newton's second law, the train's mathematical model of passing over a neutral section can be described as follows:

$$
\begin{aligned}
v \frac{d v}{d s} & =\xi\left(-\omega_{0}(v)-g(s)\right) \\
\frac{d t}{d s} & =\frac{1}{v}
\end{aligned}
$$

where $\xi$ is the rotary mass coefficient, $v, t, s$ represent, respectively, the train speed, running time and train position, and $w_{0}(v), g(s)$ are the basic resistance and gradient resistance force applied on the train per unit mass. The model is also bound by eqn (3):

$$
v(s) \leq v^{\prime}(s)
$$

where $v(s), v^{\prime}(s)$ represent, respectively, the velocity of the train, the line speed limit.

\subsection{Analysis on a neutral section and the chasing section}

We assume that the train's speed is $v_{0}$ when entering a neutral section. After passing over the neutral section, the train runs at the speed of $v_{1}$. Figure 2 shows the loss of train speed when the train passes over the neutral section as $\Delta v_{1}$. After leaving the neutral section, the train's main circuit breaker is turned on and the train may begin to accelerate until it reaches the entering speed as $v_{0}$.

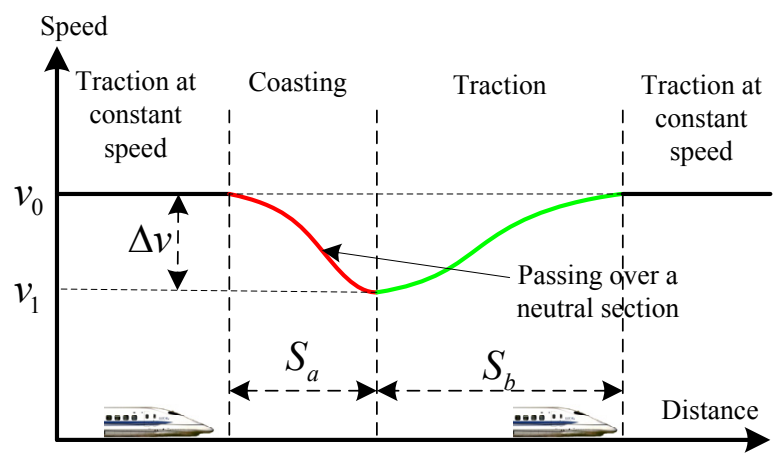

Figure 2: Loss of train speed when the train passes over a neutral section.

In Fig. 2, $S_{a}$ is the length of a neutral section, and $S_{b}$ is defined as the train's chasing distance after passing over a neutral section.

In addition, a simulation experiment is done in order to obtain a more detailed relationship among $S_{a}, S_{b}$, the loss of the train's speed and its running time.

Simulation results are shown as in Fig 3. It can be seen that the chasing distance $\left(S_{b}\right)$ doubles with the increasing of speed entering the neutral section $\left(v_{0}\right)$. When the entering speed is over $300 \mathrm{~km} / \mathrm{h}$, the chasing distance increases exponentially. Meanwhile, it can also be concluded from Fig. 3 that the delayed 
Table 1: Experiment parameters.

\begin{tabular}{|l|l|}
\hline Parameter & Value \\
\hline Train Type & CRH3C \\
\hline Ramp(\%) & 0 (plain) \\
\hline $\begin{array}{l}\text { Mode of passing over the neutral } \\
\text { section }\end{array}$ & Coasting \\
\hline Length of the neutral section & $1000 \mathrm{~m}$ \\
\hline Entering speed $(\mathrm{km} / \mathrm{h})$ & $100,150,200,250,300,350$ \\
\hline
\end{tabular}
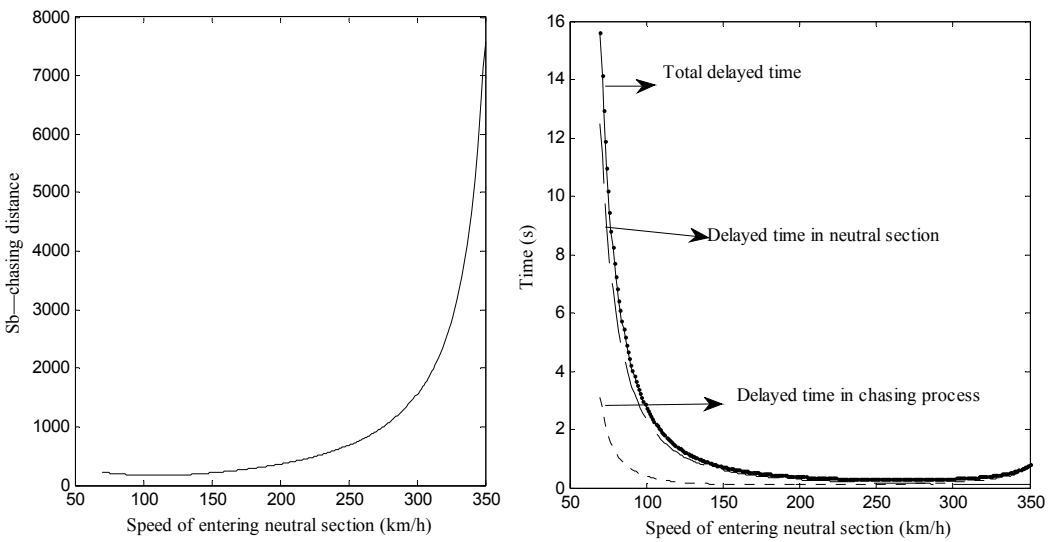

Figure 3: Chasing distance and delayed time with different entering speeds.

time augments if the speed of entering the neutral section is too low or high. However, when the speed of entering the neutral section remains at $150 \mathrm{~km} / \mathrm{h}$ $300 \mathrm{~km} / \mathrm{h}$, the total delayed time is less than $0.5 \mathrm{~s}$.

\section{Analysis on the relationship between a neutral section's layout and the train's operation state}

During the operation of a high-speed train, there are usually three operation modes; namely, traction mode, brake mode and uniform motion. Coasting is not adopted because the objects of HSR are normally on-time and high speed. Therefore, the relationship between a neutral section's layout and a train's operation state (train's speed and its running time) is analyzed mainly in three areas: traction area, uniform motion area and brake area.

\subsection{Traction area}

When a neutral section is located in a traction area, the train's speed decreases after the train enters the neutral section. After passing over the neutral section, the train begins to accelerate with its maximum traction ability until it reaches 
the current line speed limit or train's maximum allowable speed defined as the final speed. The train's speed-distance curve with passing over a neutral section is shown in Fig. 4. We assume that the train runs on a plain section. The dotted line in Fig. 4 represents the speed-distance curve without a neutral section.

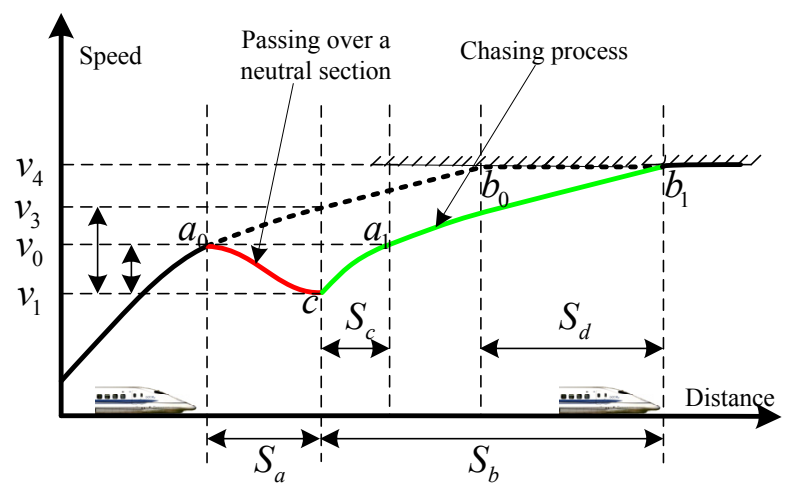

Figure 4: Automatic neutral section passing in a traction area.

$t_{a}, t_{b}, t_{c}$, and $t_{d}$ represent the costing time of a train passing over $S_{a}, S_{b}, S_{c}$, and $S_{d}$ respectively. It can also be obtained that the delayed time can be described as eqns (4) and (5).

$$
\begin{aligned}
\Delta t_{\text {total }}= & t_{a_{0} c a_{l} b_{l}}-t_{a_{0} b_{0} b_{I}} \\
= & \left(t_{a_{0} c}+t_{c a_{I}}+t_{a_{l} b_{I}}\right)-\left(t_{a_{0} b_{0}}+t_{b_{0} b_{I}}\right) \\
= & t_{a_{0} c}+t_{c a_{I}}-t_{b_{0} b_{I}} \\
& \Delta t_{\text {total }}=t_{a}+t_{c}-\frac{S_{d}}{v_{4}}
\end{aligned}
$$

where $t_{a}, t_{b}$ are only decided by the train speed of entering the neutral section under the constant length of a neutral section, and $s_{d}$ is determined by the train speed of leaving the neutral section as $v_{1}$. The speed entering a neutral section is symbolized as $v_{0}$ and the final speed is noted as $v_{4}$.

It is obvious from Fig. 5 that the closer in speed when entering a neutral section and the final speed, the less is the total delayed time. Otherwise, the delayed time will augment greatly with the increasing difference between the speed of entering a neutral section and the final speed. Therefore, it can be concluded that it is not appropriate to set up a neutral section in a traction area. 


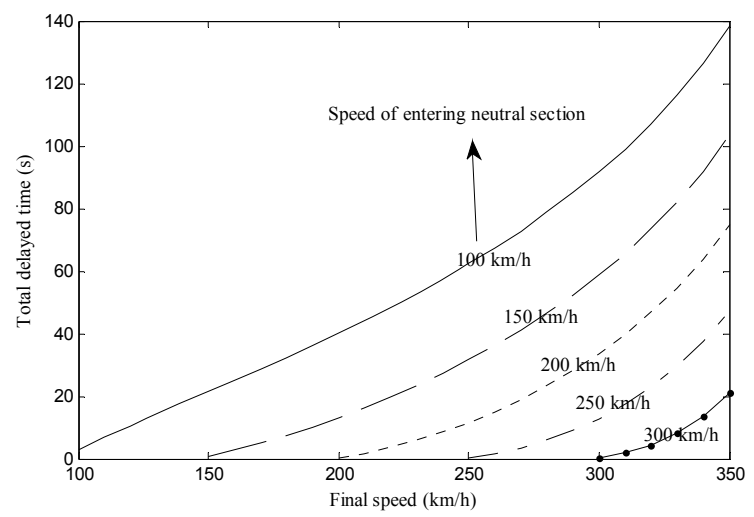

Figure 5: Delayed time with different entering speeds and different final speeds.

\subsection{Uniform motion area}

The uniform motion area is the special case of the traction area when $v_{0}$ equals $v_{4}$. As a result, eqn (6) can now be written as follows:

$$
\Delta t_{\text {total }}=t_{a}+t_{c}-\frac{S_{d}}{v_{0}}
$$

and $\Delta t_{\text {total }}$ is only decided by the constant speed $v_{0}$ under a fixed length of a neutral section. The relationship between the speed of entering a neutral section $v_{0}$ and the delayed time can be observed from Fig. 6.

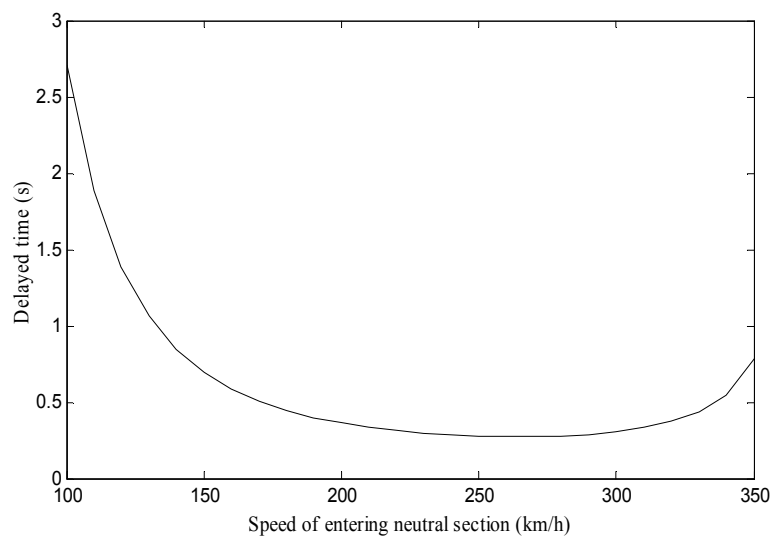

Figure 6: Delayed time with different speeds of entering a neutral section.

From Fig. 6 it can be seen that when the neutral section is in a uniform motion area, the delayed time decreases greatly. We can then conclude that it is 
quite appropriate to set up the neutral section in a uniform motion area at the speed range of $150 \mathrm{~km} / \mathrm{h}-300 \mathrm{~km} / \mathrm{h}$.

\subsection{Braking area}

When a neutral section is set up in a braking area, the train's electric brakes do not work. However, air brakes will complement the required brake force. Therefore, there will not be any loss of train speed and running time. On the other hand, when train runs in the opposite direction, the braking area then becomes the traction area. As a result, it is also inappropriate that the neutral section be in the braking area.

\subsection{Local rules of a neutral section's layout}

According to the analysis above on the relationship between a neutral section's layout and train's operation state when passing over a neutral section, local rules of a neutral section's setting can be obtained as follows.

1) Railway's up and down directions should be taken into consideration in the neutral section' s layout;

2) The neutral section should be better situated where trains run at a speed closer to the lines' limiting speed;

3) The neutral section should be better set up in an area where a train runs at a constant speed of $150 \mathrm{~km} / \mathrm{h}-300 \mathrm{~km} / \mathrm{h}$.

\section{Design of a differential evolution (DE) algorithm}

\subsection{Constraints of a neutral section's layout}

In the optimization of the layout of neutral sections, the DE algorithm's design should respect the following physical rules [3] according to the real condition of the Chinese railway infrastructure.

1) Neighboring neutral sections should be 20-30 km apart away from each other along a whole railway line;

2) A neutral section should be located at least $5 \mathrm{~km}$ away from the stop station;

3) The positions of the first neutral section and the last neutral section are bound to eqns (7) and (8).

$$
\begin{aligned}
& \delta_{1} \leq\left(l_{\text {start }}-x_{\text {start }}\right) \leq \delta_{2} \\
& \delta_{1} \leq\left(x_{\text {end }}-l_{\text {end }}\right) \leq \delta_{2}
\end{aligned}
$$

where $x_{\text {start }}, x_{\text {end }}$ denote respectively the starting point and the terminal point of the railway line, and $l_{\text {start }}, l_{\text {end }}$ represent the positions of the first neutral section and the last neutral section respectively, and $\delta_{1}, \delta_{2}$ are the minimum and maximum distances between a neutral section and the starting point as well as the endpoint of the railway line. 


\subsection{Individual agent's design and population's initialization}

We assume that the length of a neutral section is fixed and that the position of a neutral section can be represented by the middle point of the neutral section.

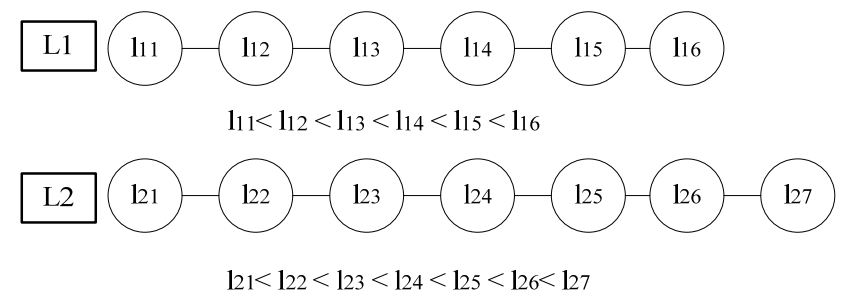

Figure 7: Individual agent's design based in order.

Thus, each agent can be described as an array with variable lengths (shown as Fig. 7).

The length of the array is the number of the neutral section on the railway line and the value of each element in the array denotes the position of each neutral section. In addition, all elements in the array are in order.

It is also defined that $L_{i}$ is the individual agent $i$ and that $l_{i j}$ represents the position of the neutral section $j$ of agent $i$, namely in mathematical, $l_{i j} \in L_{i}$. Therefore, according to the constraints of the above layout of neutral sections, the initialization rules of agent $i$ are given as follows.

1) $l_{i 1} \in\left[\delta_{1}+x_{\text {start }}, \delta_{2}+x_{\text {start }}\right]$;

2) $l_{n 1} \in\left[x_{\text {end }}-\delta_{2}, x_{\text {end }}-\delta_{1}\right]$;

3) $\forall l_{i j} \in\left\{L_{i}: j \in[2, n-1] \wedge l_{i j-1} \in\left[\delta_{2}+x_{\text {start }}, x_{\text {end }}-\delta_{2}\right]\right\}, \exists l_{i j} \in\left[l_{i j-1}+20, l_{i j-1}+30\right]$;

where $n$ is the dimension of agent, namely the number of neutral sections on the railway line. Before the optimization of neutral sections' layout, we assume that the dimension of each agent is the same according to the principle of a DE algorithm. So it would require several repetitions of the DE algorithm and optimization comparisons to find the optimal solution due to the different dimensions of the agent. Thus, we define $M P$ as the number of optimization processes or dimensions. In addition, the minimum and maximum dimension can be easily acquired on the basis of the constraints of neutral sections' layout above. To obtain enough and reliable discrete dimensions of an agent, we can pick one dimension in every five from the minimum dimension to the maximum. As a result, the number of optimization processes $M P$ is then generated.

\subsection{Cost function}

The optimization object is the minimization of the total delayed time on the whole railway line. Thus the cost function can be described as eqn (9).

$$
f=\min \left(\sum_{1}^{n} \Delta t\right)
$$




\subsection{Mutation}

For each agent $L_{i, G}$ ( $G$ is the number of iteration), the mutation vector or agent is generated as follows:

$$
v_{i, G+1}=L_{r_{1}, G}+F *\left(L_{r_{2}, G}-L_{r_{3}, G}\right)
$$

where $i \in[1, N P]$, and $N P$ is the dimensionality of the population, and $r_{1}, r_{2}, r_{3}$ are chosen at random and to be different, and $i$ should also be different with the value of $r_{1}, r_{2}, r_{3}$. So $N P$ should be more than 4 . And $F$ is called the differential weight, which varies from 0 to 2 .

\subsection{Crossover}

The crossover operation is introduced in order to increase the diversity of the interference parameter vector. The experiment vector or agent then becomes:

$$
\begin{gathered}
u_{i, G+1}=\left(u_{1, G+1}, u_{2, G+1}, \ldots, u_{n, G+1}\right) \\
u_{i j, G+1}= \begin{cases}v_{i j, G+1} \text { if randb }(j) \leq C R \text { or } j=\operatorname{rnbr}(i) \\
l_{i j, G+1} \text { otherwise }\end{cases}
\end{gathered}
$$

where $\operatorname{rand} b(j)$ is a uniformly distributed number in random between 0 and 1 for element $j$ of agent $i$, and $\operatorname{rnbr}(i)$ is a random integer between 1 and $n$ to ensure that $u_{i, G+1}$ could at least obtain one element from the mutation agent. $C R$ is called the crossover probability pre-defined by the practitioner and it varies from 0 to 1 .

\subsection{Choosing}

To determine if the experiment agent $u_{i, G+1}$ will be accepted to be part of the population, it will be compared with the object agent $L_{i, G}$ according to the DE criterion (the cost function). If the cost function has a lesser value than the experiment agent $u_{i, G+1}$, then it will be accepted. Otherwise it will simply be discarded. At each iteration, every agent will be compared with its corresponding experiment agent based on the DE criterion. And the population is thus updated until the optimal agent is found or the number of iterations is performed. However, the optimization process above is only realized for one specific dimension of an agent. Therefore, the optimization process will be repeated MP times. For each repetition, a hoped solution is generated. Finally, the candidate solution whose output of the cost function is the minimum becomes the final and optimal solution for neutral sections' layout scheme on a whole railway line.

\section{Simulation platform}

According to the DE algorithm above, a simulation platform is developed for the global optimization of neutral sections' layout scheme. The framework of the simulation can be described as in Fig. 8. And the simulation interface is shown as in Fig. 9 below. 


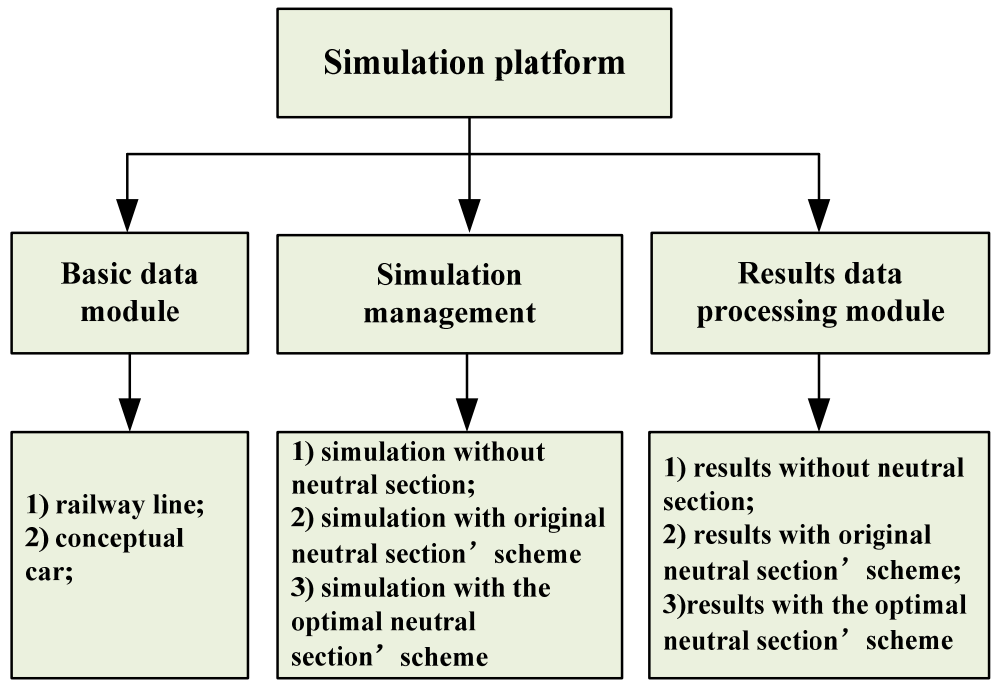

Figure 8: Framework of the simulation platform.

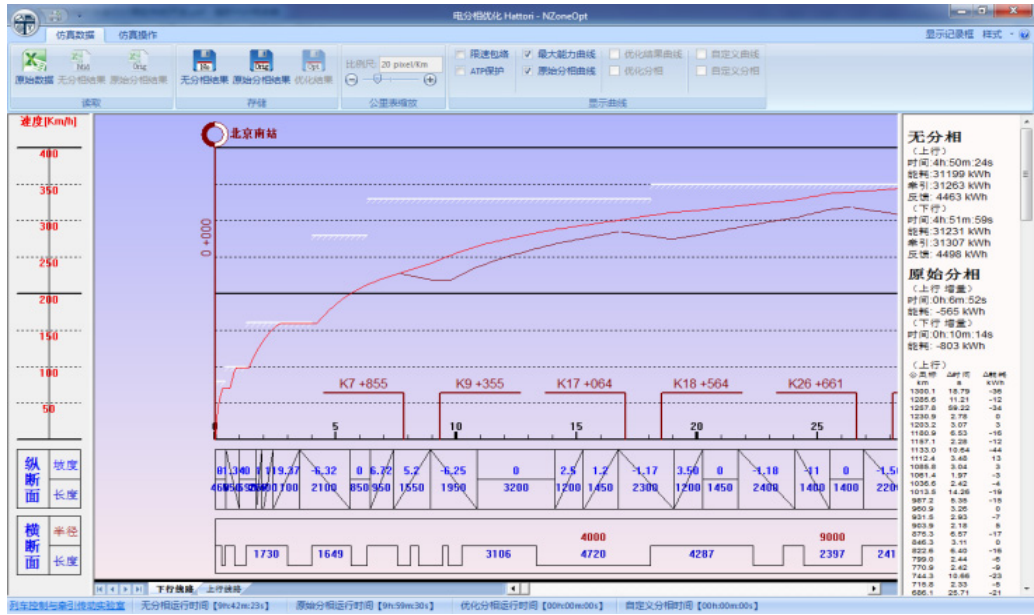

Figure 9: The simulation interface.

\section{Case study}

According to the simulation platform, several cases were studied based on the Beijing-Shanghai high speed railway line. First of all, the running time and energy consumption of the train were calculated for the whole trip with the original neutral section's layout. And then the optimal neutral section' layout scheme was obtained via the global optimization for the railway line's neutral sections' layout. Detailed results can be seen in Table 2. It can be seen that the 
running time of a train is decreased by 372 seconds with the optimal neutral sections' layout scheme.

Table 2: Optimization on neutral sections' layout for Beijing-Shanghai railway line.

\begin{tabular}{|c|c|c|c|c|}
\hline \multirow{2}{*}{$\begin{array}{l}\text { Railway } \\
\text { line } \\
\text { direction }\end{array}$} & \multicolumn{2}{|c|}{$\begin{array}{l}\text { Original neutral sections' layout } \\
\text { scheme }\end{array}$} & \multicolumn{2}{|c|}{$\begin{array}{l}\text { Optimal neutral sections' } \\
\text { layout scheme }\end{array}$} \\
\hline & $\begin{array}{l}\text { Running } \\
\text { time }\end{array}$ & $\begin{array}{l}\text { Energy } \\
\text { consumption(kWh) }\end{array}$ & $\begin{array}{l}\text { Running } \\
\text { time }\end{array}$ & $\begin{array}{l}\text { Energy consumption } \\
(\mathrm{kWh})\end{array}$ \\
\hline $\mathrm{Up}$ & 4h:57m:16s & 30634 & $4 \mathrm{~h}: 55 \mathrm{~m}: 50 \mathrm{~s}$ & 30633 \\
\hline Down & $5 \mathrm{~h}: 2 \mathrm{~m}: 13 \mathrm{~s}$ & 30428 & $4 \mathrm{~h}: 55 \mathrm{~m}: 27 \mathrm{~s}$ & 30483 \\
\hline Total & $9 \mathrm{~h}: 57 \mathrm{~m}: 29 \mathrm{~s}$ & 61062 & $9 \mathrm{~h}: 51 \mathrm{~m}: 17 \mathrm{~s}$ & 61116 \\
\hline
\end{tabular}

\section{Conclusion}

In this paper, a train's operation is first analysed in detail when passing over a neutral section. According to train traction calculation regulation, the mathematical model of passing through a neutral section is then established. What's more, the relationship is studied between a neutral section's layout and a train's operation state. Global optimization of neutral sections' layout scheme is then realized when a DE algorithm is introduced. A simulation platform for neutral sections' layout scheme optimization is finally developed. Via simulation experiments, the DE algorithm is proven to be correct and effective.

\section{References}

[1] B. S. Liu, Q. Y. Wang. Study on Velocity and Time Loss of the Electric Locomotive Passing a Neutral section. Electrical Engineering, pp. 16-18, 2010 .

[2] L. Tang. Influence on the Electric Multiple Unit Running Speed \& Time with Electric Phrase Separation Settlement. Journal of Transportation Engineering and Information, 4(9), pp. 63-68, 2011.

[3] P. F. Sun. Research on Relationship between Optimizing Scheme of Neutral Section and Train Running Time, Chengdu Southwest Jiaotong University, 2012.

[4] Y. Y. Sheng. Development Trend of the Scheme of Auto-Passing Phase Separations for Electric Locomotive and Emus. Electric Drive for Locomotive, 6, pp. 7-10, 2004.

[5] C. Z. Chen. The Analysis on the Course of Electric Locomotive Passing through Phase Separation, Chengdu Southwest Jiaotong University, 2007.

[6] D. M. Ma. Theoretical Analysis of the Transient Process While Locomotive/EMU Passing Electric Phase Break, Chengdu Southwest Jiaotong University, 2008.

[7] J. L. Zhu, H. C. Li and Q. Y. Wang, Optimization Analysis on the Energy Saving Control for Trains, Journal of the China Railway Society, 29(2), pp. 104-108, 2008. 